\title{
Non-GPS Navigation with the Personal Dead-Reckoning System
}

\author{
Lauro Ojeda and Johann Borenstein \\ The University of Michigan, 2260 Hayward Street, Ann Arbor MI 48109, USA \\ lojeda@umich.edu, johannb@umich.edu
}

\begin{abstract}
This paper introduces a positioning system for walking persons, called "Personal Dead-reckoning" (PDR) system. The PDR system does not require GPS, beacons, or landmarks. The system is therefore useful in GPS-denied environments, such as inside buildings, tunnels, or dense forests. Potential users of the system are military and security personnel as well as emergency responders.

The PDR system uses a small 6-DOF inertial measurement unit (IMU) attached to the user's boot. The IMU provides rate-of-rotation and acceleration measurements that are used in real-time to estimate the location of the user relative to a known starting point. In order to reduce the most significant errors of this IMU-based system — caused by the bias drift of the accelerometers-we implemented a technique known as "Zero Velocity Update" (ZUPT). With the ZUPT technique and related signal processing algorithms, typical errors of our system are about $2 \%$ of distance traveled. This typical PDR system error is largely independent of the gait or speed of the user. When walking continuously for several minutes, the error increases gradually beyond 2\%. The PDR system works in both 2-dimensional (2-D) and 3-D environments, although errors in Z-direction are usually larger than $2 \%$ of distance traveled.

Earlier versions of our system used an impractically large IMU. In the most recent version we implemented a much smaller IMU. This paper discussed specific problems of this small IMU, our measures for eliminating these problems, and our first experimental results with the small IMU under different conditions.
\end{abstract}

Keywords: Personal Odometry, Personal Dead-reckoning, non-GPS navigation, GPS-denied navigation, Inertial Measurement Unit, IMU

\section{INTRODUCTION}

This paper describes our Personal Dead-reckoning (PDR) system for measuring and tracking the momentary location and trajectory of a walking person, even if GPS is not available. Such a system is of value for military and security personnel, as well as for emergency responders. For example, our system would allow tracking the position of soldiers in urban combat operations. Another application involves the "clearing" of a large building by emergency or security personnel. Their challenge often is to keep track of rooms already cleared and areas that were not cleared, yet. Our system's ability to track each person's location provides a useful solution for this problem. Other applications for the PDR system are the interior mapping of buildings and improved situation awareness for soldiers.

As mentioned, our proposed PDR system does not require GPS. This is an important distinction, since GPS is not available indoors. Furthermore, GPS is unreliable under dense foliage, in so-called "urban canyons," and generally in any environment, in which a clear view of a good part of the sky is not available.

There are some other approaches to personal position estimation without GPS. Typically, these other systems require external references, also called "fiducials," which in most cases must be installed in the work space at precisely surveyed locations before the system can be used. This installation is time consuming and expensive, and in the cases of emergency response or urban combat completely unfeasible. Some fiducial-based systems also require an active radiation source, such as infrared light [1], ultrasound [2], or magnetic fields [3], which may be undesirable in security-related applications. Generally, fiducial-based systems perform well and are able to provide absolute position and orientation in 
real-time. If the application permits the installation of fiducials ahead of time, then these systems have the significant advantage that errors don't grow with time, whereas with our system they do.

Another way of implementing absolute position estimation is computer vision [4] [5]. Images are compared and matched against a pre-compiled database. Computer vision has the advantage that the environment does not need to be modified, but the approach requires potentially very large databases. Work is also being done on so-called Simultaneous Location and Mapping (SLAM) methods, which don't require a precompiled database [6]. However, SLAM systems are not as reliable, may accrue errors over time and distance, and poor visibility and unfavorable light conditions can result in completely false position estimation.

The scientific literature offers a few approaches that do not require external references. The simplest one of them is the pedometer, that is, a device that counts steps. Pedometers must be calibrated for the stride length of the user and they produce large errors when the user moves in any other way than his or her normal walking pattern. One commercially available personal navigation system based on this principle is the Dead Reckoning Module (DRM) [7]. The DRM uses accelerometers to identify steps, and linear displacement is computed assuming that the step length is constant. Heading is measured using a digital compass, which is combined with the traveled distance (step counts) to estimate 2-D position. Under this condition, Pointresearch/Honeywell claims an accuracy of up to $5 \%$ of the traveled distance. However, we believe that the requirement for a constant step length is impractical, since step size changes as a function of operational needs, fatigue, and weight carried by the user.

A very sophisticated pedometer-like approach was introduced by Cho and Park [2]. His system uses a two-axes accelerometer and a two-axes magnetometer located on the user's boot. Step length is estimated from accelerometers readings that are passed through a neural network, and advanced Kalman Filter techniques are aimed at reducing the effect of magnetic disturbances. While the reported results in an outdoor environment are very good, we found that indoors, especially in large steel structures, magnetic disturbances are omnipresent and varying, making it virtually impossible to filter them out.

Other solutions actually measure the length of every stride in real-time. One such solution using ultrasonic sensors attached to the user's boots is explained in [8]. Ultrasonic sensors require a direct line of "sight" between the boots, which may be a problem on rough terrain. In straight-line walking experiments the authors report an average and maximum error of $1.3 \%$ and $5.4 \%$, respectively. Another approach measures the RF phase change between a reference signal located in a waist pack and the one coming from a transmitter located on each boot [9]. A drawback of these approaches is that position estimation is restricted to 2-D environments since neither system can determine altitude changes and assumes that any change is horizontal. Another potential problem is that these technologies use active emissions, which are undesirable for military applications, and they are vulnerable to external interference from the environment or from other units.

In our own previous work we demonstrated accurate 3-D position estimation using a six-Degree-of-Freedom (6-DOF) Inertial Measurement Unit (IMU) attached to the user's boot [10] [11]. The main disadvantage of our previous PDR system (shown in Figure 1a), was that our IMU, the SiIMU01 made by BAE [12] was bulky, heavy and expensive. In this paper we present our new PDR system (shown in Figure 1b), which uses the much smaller and lighter nano IMU ("nIMU," in short) made by MemSense [13]. The nIMU, however, has significantly worse performance specifications than the SiIMU01.

Both the old and the new version of our system compute the complete trajectory of the boot during each step. On first glance it appears that this approach is destined to fail, since measuring linear displacement using accelerometers is not very feasible. That is because data samples from accelerometers must be integrated twice to yield linear displacement and this process tends to amplify even the smallest errors, notably those due to bias drift. However, we use a practical method (explained in detail in Section 3.2) that almost completely eliminates this problem - under certain operational conditions. We found that such operational conditions exist in legged motion, such as when people walk, run, or even climb. Conversely, our method does not work at all with wheeled, sea-, or airborne motion. We should also note that since the PDR system uses only passive sensors, it has a zero-radiation signature, i.e., it does not emit any signals. This makes our system "invisible" to sensors in hostile environments and immune to interference or jamming.

The PDR system offers two distinct capabilities: (1) it can measure linear displacement (i.e., odometry) and (2) it can estimate the subject's actual location in terms of $x, y$, and $z$ coordinates, relative to a known starting location. Such a function, if performed without external beacons or landmarks, is referred to as "dead-reckoning." The simpler but less useful function - Odometry - has errors of less that $2 \%$ of distance traveled, regardless of the duration of the walk. The 
more complex but also more useful dead-reckoning function produces errors of up $2 \%$ of distance traveled in walks of up to about two minutes. Longer walks will produce larger errors due to bias drift in the gyroscopes. The bias drift errors in the gyroscopes can be measured and removed from subsequent readings when the user stands still for a few seconds. However, any accumulated heading error remains.

\section{PDR SYSTEM HARDWARE}

Our current system uses a small IMU strapped to the side of the subject's foot, as was shown in Figure 1. The IMU is connected to a tablet-style laptop computer through an RS-422 communication port. The IMU is powered using a small external 7.8-Volt Lithium Polymer battery, making the whole system portable. The computer runs the Linux operating system patched with a real-time extension [14] and our algorithm runs in real-time.

\subsection{IMU Characterization}

Our earlier PDR system used the BAE SiIMU01 IMU. This IMU has excellent performance characteristics, but it is too bulky and heavy for practical applications. In the present work we implemented the MemSense nano IMU. This device is very small and lightweight, but its performance is much worse than that of the SiIMU01. The small size of the nIMU has the advantage that it can be easily attached to a boot and perhaps even fit in the sole of a boot. Table 1 compares the two units side-by-side.

Before putting the nIMU to use, we performed a series of experiments to characterize the deterministic and non-deterministic errors of each of its three gyroscopes.

\subsection{Deterministic Errors}

Among the deterministic errors, the non-linearity of the scale factor and scale factor sensitivity to changes in temperature are the most significant ones. These deterministic errors can be modeled as a family of third-order polynomial functions. Once the deterministic errors are modeled, they can be compensated for by applying an appropriate set of third-order polynomial compensation functions, as shown in Figure 2 for one of the three gyros of our nIMU. Much greater detail on this highly effective compensation method is provided in our earlier publication [15].

Table 1: Key specifications of the large SiIMU and

\begin{tabular}{|l|r|r|}
\hline & $\begin{array}{c}\text { BAE } \\
\text { SilMU01 }\end{array}$ & $\begin{array}{c}\text { Memsense } \\
\text { nIMU }\end{array}$ \\
\hline Weight (gram) & 250 & 15 \\
\hline Size (mm) & $75 \times 75 \times 45$ & $45 \times 23 \times 13$ \\
\hline Bandwidth (Hz) & 75 & 50 \\
\hline \multicolumn{3}{|c|}{ Gyroscopes } \\
\hline Range (deg/sec) & $\pm 1,000$ & $\pm 1,200$ \\
\hline $\begin{array}{l}\text { Angle Random } \\
\text { Walk (deg/rt-hr) }\end{array}$ & 1.0 & 4.4 \\
\hline Bias drift (deg/hr) & 5.0 & 80 \\
\hline \multicolumn{3}{|c|}{ Accelerometers } \\
\hline Range (g) & \pm 50 & \pm 10 \\
\hline
\end{tabular}
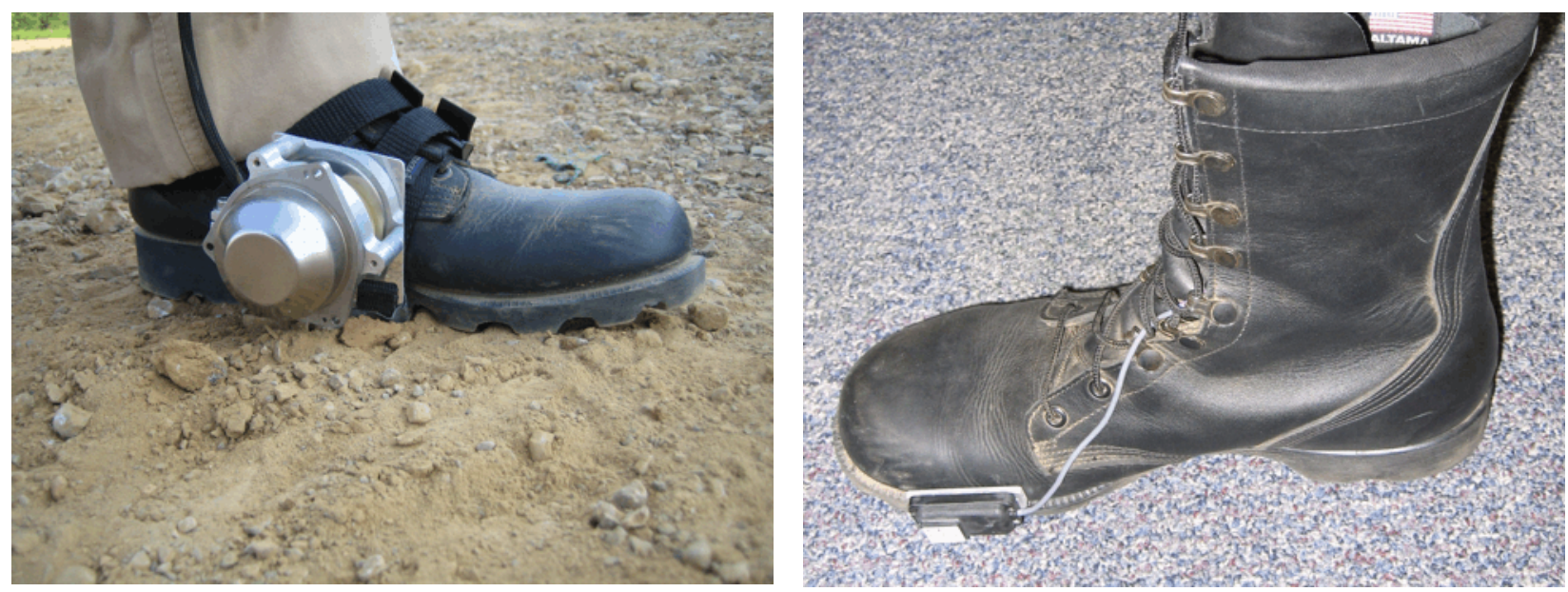

Figure 1: The University of Michigan's two IMUs mounted to boots. (a) The SiIMU used in our earlier proof-of-concept prototype; (b) the small-sized nIMU, described in this paper. 


\subsubsection{Acceleration-Dependent Bias}

Coriolis-based gyroscopes are sensitive to acceleration changes, and in the case of the nIMU, the bias coefficient is indeed affected to a significant degree by linear accelerations. Given that during typical walking the IMU is constantly subjected to varying linear accelerations, this phenomenon introduces significant errors in the attitude computation.

During walking, linear accelerations are produced in two ways:

1. As the foot goes through the various phases of taking one step forward, it is subjected to linear accelerations. During regular walking, these accelerations are on the order of $2 \mathrm{~g}$, mostly in the forward direction, and, to a smaller degree, in the vertical direction.

2. The gravity vector produces varying linear accelerations along all three principal axes of the IMU's coordinate frame, since the boot continuously changes its attitude during walking.

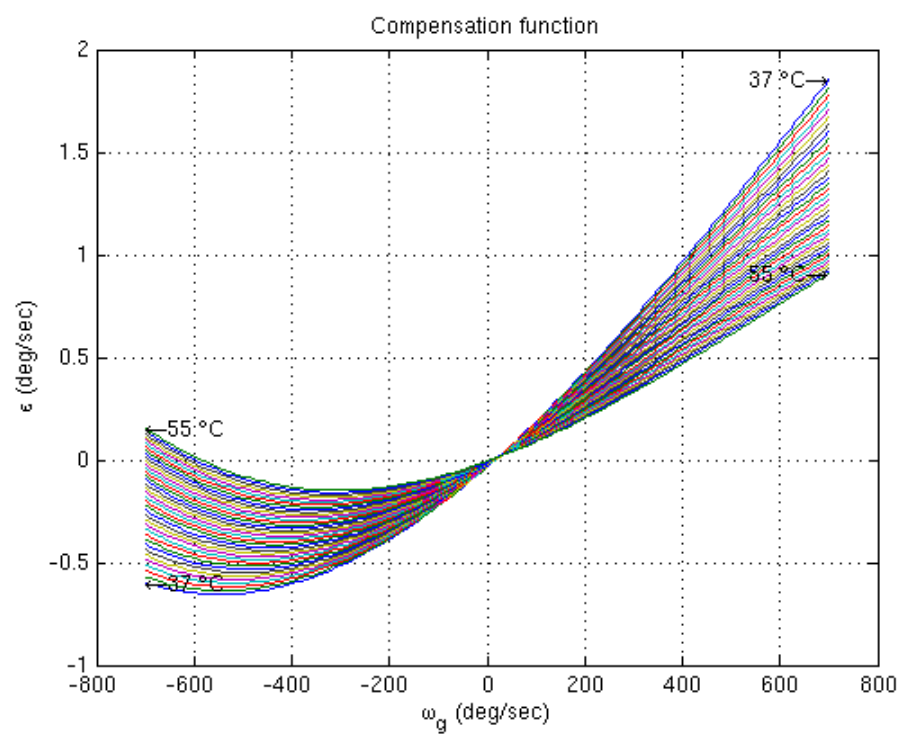

Figure 2: MemSense nIMU scale factor non-linearity and sensitivity to changes with temperature.

The unwanted effect of the gravity vector on the rate measurements of the gyros is relatively easy to measure and correct. In a first attempt we focused on correcting the heading error. Since heading is mostly affected by the z-axis gyro measurements, denoted $\omega_{z}$, and because most of the acceleration changes will occur along the forward direction $\left(a_{x}\right)$, we measure the acceleration that the gyros are subject using the IMU's accelerometers and we apply bias correction $\left(B_{g x}\right)$ as follows:

$$
\omega_{z}=\omega_{z o}-a_{x} B_{g x}
$$

where

$\omega_{z o}-\mathrm{z}$-axis angular rate before compensation

$\omega_{z}-\mathrm{z}$-axis angular rate after applying acceleration compensation

In a first approximation, we determined the correction coefficient $B_{g x}$ experimentally using the gravity component as a constant source of acceleration.

\subsection{Non-Deterministic Errors}

To measure non-deterministic errors, we performed the Allan variance analysis as described in [16] to determine the bias instability and Angle Random Walk (ARW) of the gyroscopes. The estimated bias instability parameter for the gyroscopes was about $80 \mathrm{deg} / \mathrm{sec}$ (see Figure 3), which represents the estimated angle error per unit of time due to variation of the bias error. The ARW error contribution for each of the gyros was $4.4 \mathrm{deg} \cdot \mathrm{hr}^{-1 / 2}$. This error is the angle error introduced by the integration of noise over time.

The non-deterministic ARW and bias instability errors cannot be modeled and compensated for with deterministic compensation functions; they can be modeled only in statistical terms. In order to reduce

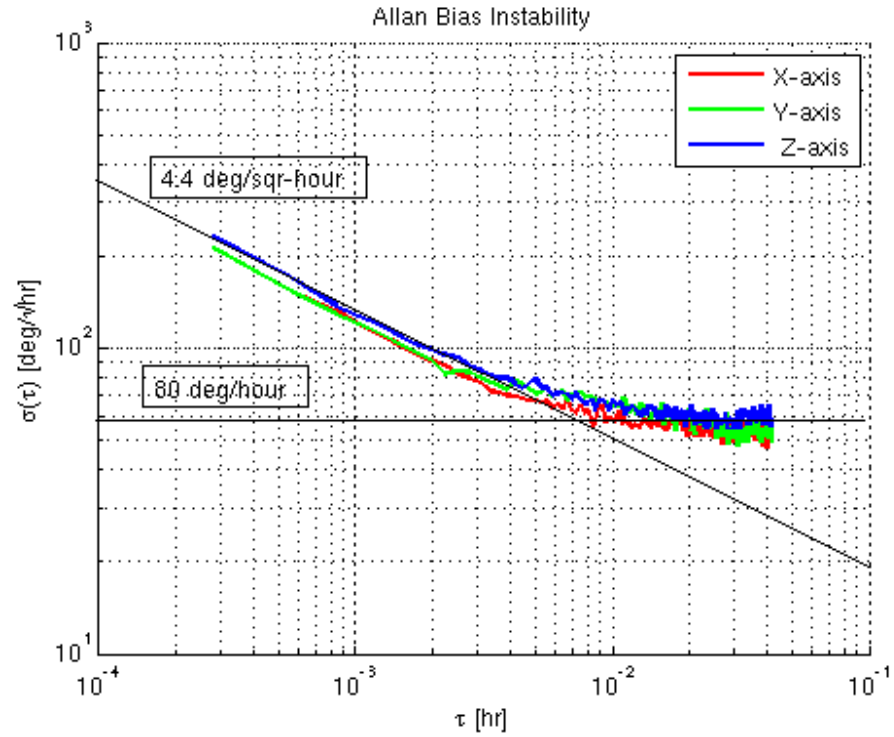

Figure 3: Allan variance analysis for our MemSense nIMU. 
or counteract these errors, additional sensors may be needed with error characteristics that are complementary to those of the nIMU's gyros. For example, in outdoor environments, magnetometers can be used effectively to bound the nondeterministic errors of the gyros, although we have not yet done this in our system. A limitation is that magnetometers are virtually unusable in steel structures and many other indoor environments. Another alternative for reducing errors caused by the bias drift of the gyros is to detect periods of zero motion (i.e., when the user is standing still) and update the gyro bias drift during those periods.

\section{PDR SYSTEM SOFTWARE}

The software for the PDR system has three modules:

- Position estimation module

- Zero Velocity Updates” module (ZUPT)

- Step detection module

These modules are explained in more detail in the remainder of this section.

\subsection{Position Estimation}

In this section we give a brief summary of the navigation equations used in our system. For a more detailed explanation see [17].

We follow the convention used in aeronautics for the designation of the navigation and body frames. In mobile robotics, the so-called Euler equations are commonly used for attitude representation. However, Euler equations have singularities at $\pm 90^{\circ}$ - a limitation that is irrelevant in most ground-based mobile robot applications. However, since in our application the IMU is attached directly to the boot of a walking or running person, tilt angles of $90^{\circ}$ or more are possible and likely. For this reason we chose the Quaternion representation, which handles any tilt angles.

The Quaternion, $q$, is a vector that defines attitude using four parameters, $a, b, c$ and $d . q$ propagates as a function of the body angular rates, $\omega_{b}$, according to:

$$
\dot{q}=\frac{q \cdot p}{2}
$$

where $p=\left[0, \omega_{b}\right]$ and $\omega_{b}=\left[\omega_{x}, \omega_{y} \omega_{z}\right]$.

Once attitude is computed, the body acceleration, $a_{b}$, can be computed in terms of the navigation reference frame, $a_{n}$, using the quaternion vector

$$
a_{n}=q a_{b} q^{*}
$$

where $q^{*}=(a-b-c-d)$ is the complex conjugate of $q$.

In order to minimize the errors associated with the digital implementation of these algorithms, we used optimized discrete-time algorithms as explained by Savage [18].

Velocity, $v_{n}$, can be computed by integrating the accelerations in the navigation frame after eliminating the local gravity component $g_{l}$

$$
v_{n}=\int \dot{v}_{n} d t=\int\left(a_{n}+g_{l}\right) d t
$$

Finally, position can be computed as the integral of the velocity over time

$$
p_{n}=\int v_{n} d t
$$




\subsection{Zero Velocity Updates (ZUPT)}

Figure 4 shows some of the phases of a stride during normal walking. As is evident from the motion sequence, Point A on the bottom of the sole is in contact with the ground for a short portion of time, $\Delta T . \Delta T$ lasts roughly from just before Midstance $\left(T_{1}=0.48 \mathrm{sec}\right)$ to just after Terminal Stance $\left(T_{2}=\right.$ $0.72 \mathrm{sec}$ ) and is $\sim 0.24 \mathrm{sec}$ in the example here (terminology based on [19]). During that time and unless the sole is slipping on the ground, ' $\mathrm{A}$ ' is not moving relative

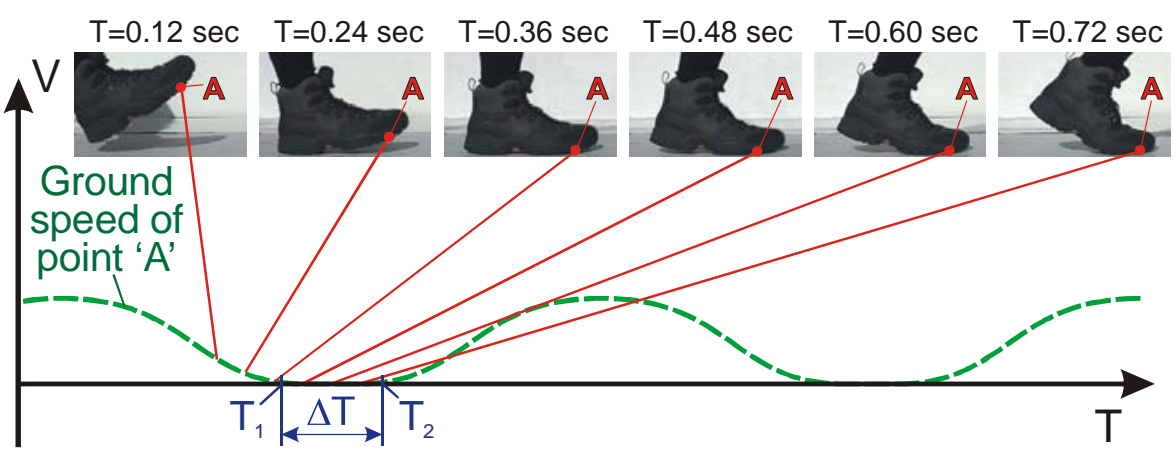

Figure 4: Key phases in a stride. During $\Delta T$, all velocities and accelerations of point $\mathrm{A}$ in the sole of the boot are zero.

to the ground and the velocity vec-

tor of ' $A$ ' is $\boldsymbol{V}_{A}=0$. The non-slip assumption is warranted because during that phase almost all of the body's weight rests solely on the area of the sole around 'A', thereby increasing traction.

Since the condition $\boldsymbol{V}_{A}=0$ is maintained for the significant period of time $\Delta T$ and not just for an instance, we reason that at least sometime during $\Delta T$ the velocity vector of Point $\mathrm{A}$ is also zero. We expect the three velocities to show readings of zero during this time. If the reading is not zero, then we assume that the difference between zero and the momentary reading is the result of accumulated errors during the step interval. It is now trivial to reset the velocity error to the known zero condition. This way we can effectively remove the accumulated errors from the accelerometer output, at least for a few seconds. Luckily, it is the nature of walking or running that the next footstep is just a second away, allowing us to repeat this cycle over and over without accumulating significant errors. This frequent resetting of velocities to the known and absolutely true value of zero assures that any error produced during one step is not carried over to the next ones. For example, if the subject's foot actually slipped during one step, then the resulting error in velocities exists for just the duration of this one step. Subsequent steps are again error-free. The resulting error in position is just a few centimeters and it remains constant for the remainder of the walk, unless new errors occur.

In the scientific literature, this method of counteracting drift is called "Zero Velocity Update" (ZUPT) and is commonly used in underwater navigation [20]. ZUPT is also used in oil drilling, where it provides real-time monitoring of the position and orientation of the bottom hole assembly [21]. In these applications, ZUPT has been used successfully with update intervals between two to ten minutes depending on the quality of the sensors. The accuracy of the ZUPTbased solution depends on the time interval between ZUPT points. As mentioned, for walking or running these conditions occur once on every footfall, that is, about once every second. A detailed explanation of the ZUPT method can be found in [22].

Figure 5 shows the computed velocities during a few strides of a subject walking at walking speed. Note how quickly the uncorrected velocities (interrupted green line) diverge from ground truth, which should be zero in all directions during a period of time during each step.

The elegance of this approach lies in the fact that in each stride we know at least once the true velocity and acceleration of Point A. Our knowledge of the velocity and acceleration being zero and the resulting ZUPT correction is always absolute, not relative to the previous correction. Therefore, at least once during every step the accumulated errors can be removed or bounded.

\subsection{Step Detection}

For the ZUPT algorithm to work properly, it is not necessary to identify correctly the exact onset and end of $\Delta T$. Rather, it is sufficient to identify a single instance, $T_{s}$, within $\Delta T$, in which all accelerations and velocities are zero. In practice, this is not trivial, because the accelerometers suffer from drift, so they never show zero exactly. Experimentally we found that the best indication for $T_{s}$ can be obtained by observing the three components $\left(\omega_{x}, \omega_{y}, \omega_{z}\right)$ of the angular velocity vector, $\omega$. During $\Delta T$, the absolute values of these components have a local minimum and their absolute value is 
small (close to zero). Of course, $\omega$ is directly measured by the three gyroscopes of the IMU, so that data is readily available.

We implement these two empiric rules in our algorithm as follows:

1. The gyro signals, $\omega_{b}$, are divided in segments of $n=100$ samples, which correspond to 0.5 seconds worth of data. The exact size of the segment is not critical, but for best results the segment should be short and comparable to the duration of the fastest step. This assures that there is at least one period $\Delta T$ in each segment.

In each segment we compute an array of $n=100$ scalars, $\omega_{s}$. Each element in $\omega_{s}$ is a scalar representing the amplitude of the $\omega_{b}$ for that sample.

$$
\omega_{\mathrm{s}, \mathrm{i}}=\sqrt{\omega_{\mathrm{x}, \mathrm{i}}^{2}+\omega_{\mathrm{y}, \mathrm{i}}^{2}+\omega_{\mathrm{z}, \mathrm{i}}^{2}}
$$

2. Next we determine which elements of $\omega_{s}$ are smaller

than a certain threshold, $\Omega$. We copy all elements that meet this test into a new array, $\omega_{T}$

$$
\omega_{T, i}=\left\{\begin{array}{ccc}
\omega_{s, i} & \text { for } & \omega_{s, i}<\Omega \\
K & \text { for } & \omega_{s, i} \geq \Omega
\end{array}\right.
$$
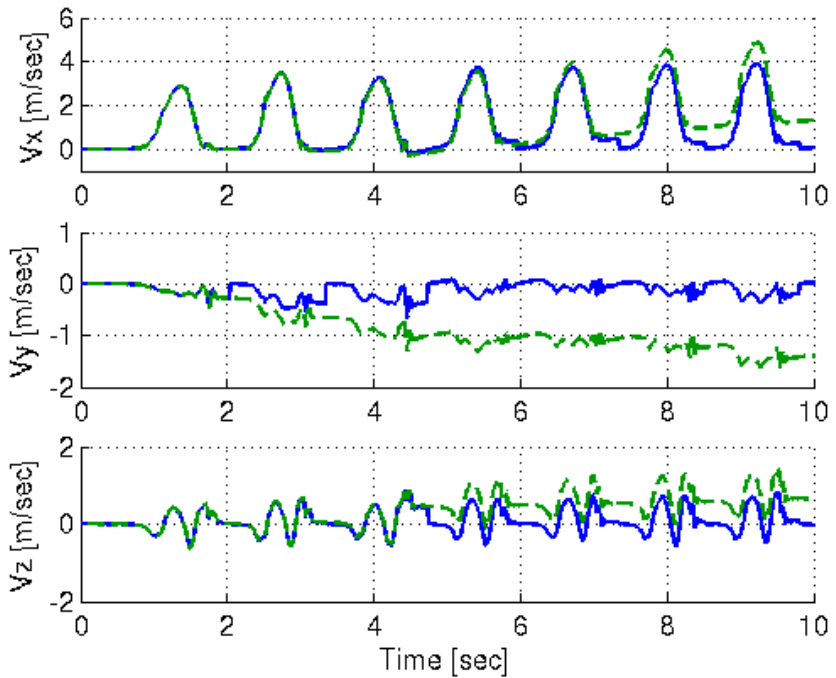

Figure 5: Linear velocity of the boot. Dotted green line: without forward.

where $K$ is some large number. If all elements $\omega_{T, I}=K$, then we conclude that there was no period $\Delta T$ in this segment of $n=100$ samples and we investigate the next segment. If there are one or more $\omega_{T, i} \neq K$ in a given segment, then we search for the smallest one and denote the time associated with this sample $T_{s}$. $T_{s}$ is the instance, at which the locally and absolutely smallest rotation was measured and for ZUPT it signifies the instance, at which all accelerations and velocities of Point A should have been zero.

In practice we found that in rare cases this algorithm may detect false footfalls when the foot is actually in mid-air and its total rate of rotation is lower than the threshold $\Omega$. In order to eliminate this ambiguity, we are currently implementing an enhanced detection technique that confirms a footfall by looking at accelerometer data.

After applying the Step Detection and ZUPT algorithm, there is an additional stage of conditioning the sensor data. A detailed discussion of this stage is beyond the scope of this paper. The experimental results of the following section, however, reflect the application of the additional data conditioning stage.

\section{EXPERIMENTAL RESULTS}

We performed several experiments with our system, most of them involving 2-D closed-loop tests at different paces. The last experiment described in this section had some elevations, but they were not significant enough to consider the walks as 3-D.

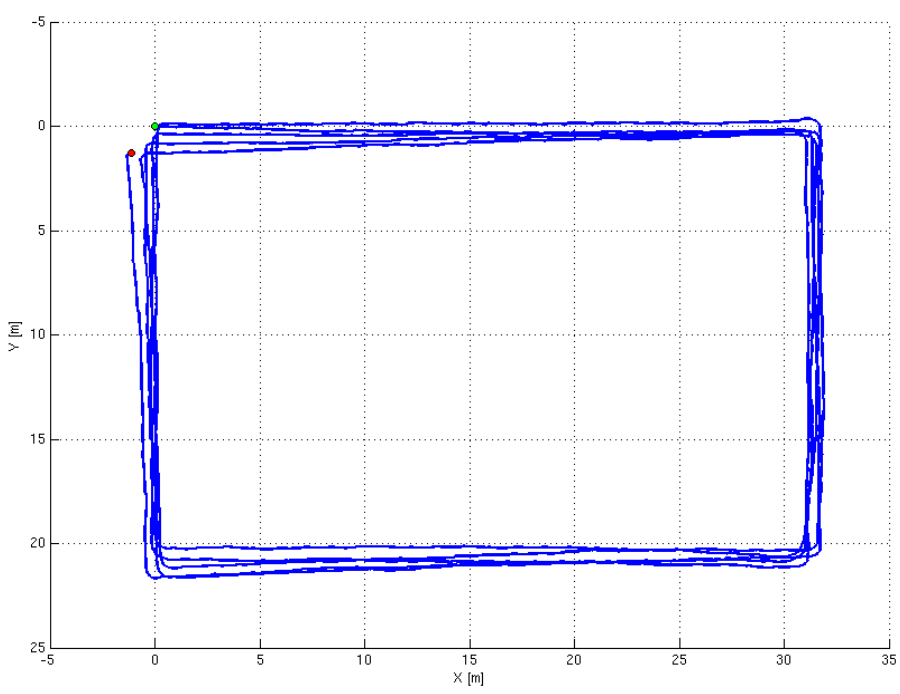

Figure 6: Trajectory of a subject walking along the 2-D rectangular closed-loop path. 


\subsection{Short Walk}

In this experiment the subject walked along a rectangle-shaped path (Figure 6). The longer side of the rectangle was just over 30 meters in length, and the shorter one about $20 \mathrm{~m}$, resulting in a total path length of $D=104 \mathrm{~m}$. We ran five experiments in clockwise (CW) and five experiments in counter-clockwise (CCW) direction. In all cases the subject walked at the normal walking pace of $1 \mathrm{~m} / \mathrm{sec}$. After completing each loop, the subject stood still for about $30 \mathrm{sec}$. This zero motion condition was detected by our software, which updated the bias drift using the new information. The same experiment was performed by five different subjects.

The absolute return position error in the $\mathrm{x}-\mathrm{y}$ plane was computed as

$$
E_{a}=\sqrt{x_{e}^{2}+y_{e}^{2}}
$$

where

$x_{e}$ - return position error in X-direction.

$y_{e}$ - return position error in Y-direction.

We also computed the relative average error, $E_{r}$, which expresses the average error as a percentage of total travel distance, $D$

$$
E_{r}=100 \frac{E_{a}}{D}
$$

Figure 7 shows the final position errors for these runs. Averages of the results of these runs are summarized in Table 2 and Table 3.

We also performed a second set of experiments under similar conditions. This time the subject walked as fast as possible, but without running. The average walking speed was 1.8 $\mathrm{m} / \mathrm{sec}$. Figure 8 shows the final position errors for these ten walks and numeric results are summarized in Table 4 and Table

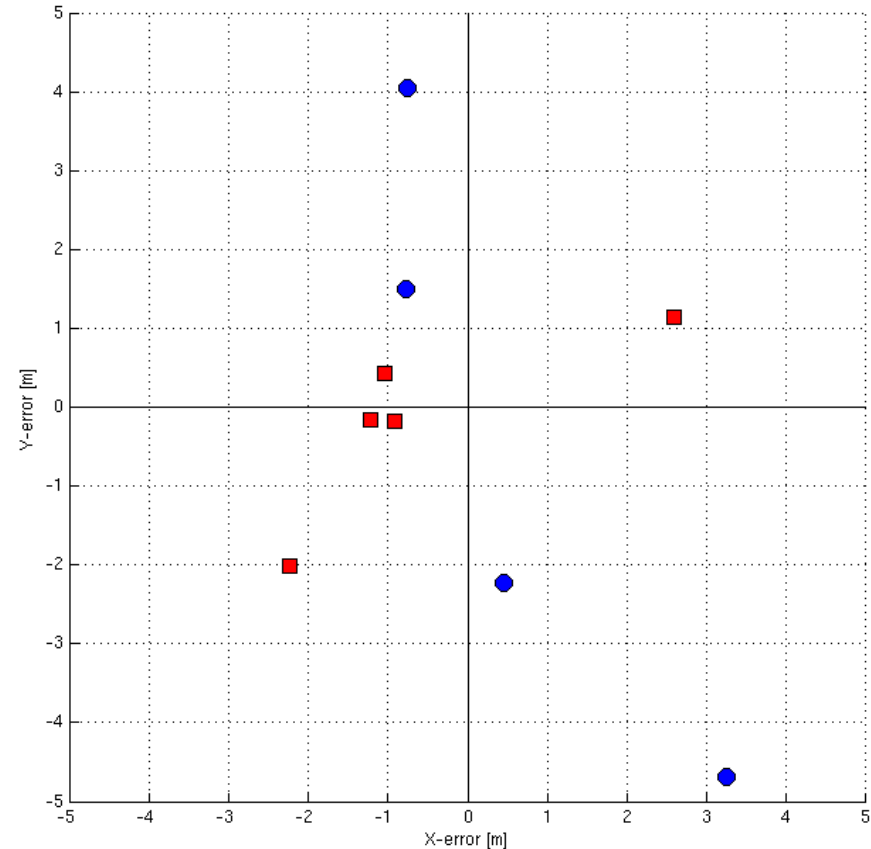

Figure 7: Return position errors in the vicinity of the target point $(0$ 0 ) after walking normally along the 2-D closed-loop path in either (O-blue color) CW or ( $\square$-red color) CCW direction.
Table 2: Summary of return position errors for the CW 2-D closed-loop path experiment (normal walk).

\begin{tabular}{|l|c|r|c|c|c|}
\hline & $\begin{array}{c}X_{e} \\
{[\mathbf{m}]}\end{array}$ & $\begin{array}{c}Y_{e} \\
{[\boldsymbol{m}]}\end{array}$ & $\begin{array}{c}\boldsymbol{D} \\
{[\mathbf{m}]}\end{array}$ & $\begin{array}{c}E_{a} \\
{[\boldsymbol{m}]}\end{array}$ & $\begin{array}{c}E_{r} \\
{[\%]}\end{array}$ \\
\hline Walk 1 & -0.77 & 1.5 & 524.2 & 1.69 & 0.32 \\
\hline Walk 2 & -0.75 & 4.04 & 524.3 & 4.11 & 0.78 \\
\hline Walk 3 & 1.94 & -5.12 & 519.5 & 5.48 & 1.05 \\
\hline Walk 4 & 3.26 & -4.68 & 525.0 & 5.7 & 1.09 \\
\hline Walk 5 & 0.46 & -2.23 & 511.9 & 2.28 & 0.44 \\
\hline Average & & & $\mathbf{5 2 0 . 9}$ & $\mathbf{3 . 8 5}$ & $\mathbf{0 . 7 4}$ \\
\hline
\end{tabular}

Table 3: Summary of return position errors for the CCW 2-D closed-loop path experiment (normal walk).

\begin{tabular}{|l|c|c|c|c|c|}
\hline & $\begin{array}{c}\boldsymbol{X}_{e} \\
{[\mathbf{m}]}\end{array}$ & $\begin{array}{c}Y_{e} \\
{[\mathbf{m}]}\end{array}$ & $\begin{array}{c}\boldsymbol{D} \\
{[\mathbf{m}]}\end{array}$ & $\begin{array}{c}\boldsymbol{E}_{\mathbf{a}} \\
{[\mathbf{m}]}\end{array}$ & $\begin{array}{c}\boldsymbol{E}_{r} \\
{[\%]}\end{array}$ \\
\hline Walk 1 & -1.05 & 0.43 & 531 & 1.13 & 0.21 \\
\hline Walk 2 & -0.92 & -0.17 & 527 & 0.94 & 0.18 \\
\hline Walk 3 & -1.22 & -0.16 & 522 & 1.23 & 0.24 \\
\hline Walk 4 & 2.59 & 1.14 & 524 & 2.83 & 0.54 \\
\hline Walk 5 & -2.24 & -2.01 & 507 & 3.01 & 0.59 \\
\hline Average & & & $\mathbf{5 2 2}$ & $\mathbf{1 . 8 3}$ & $\mathbf{0 . 3 5}$ \\
\hline
\end{tabular}




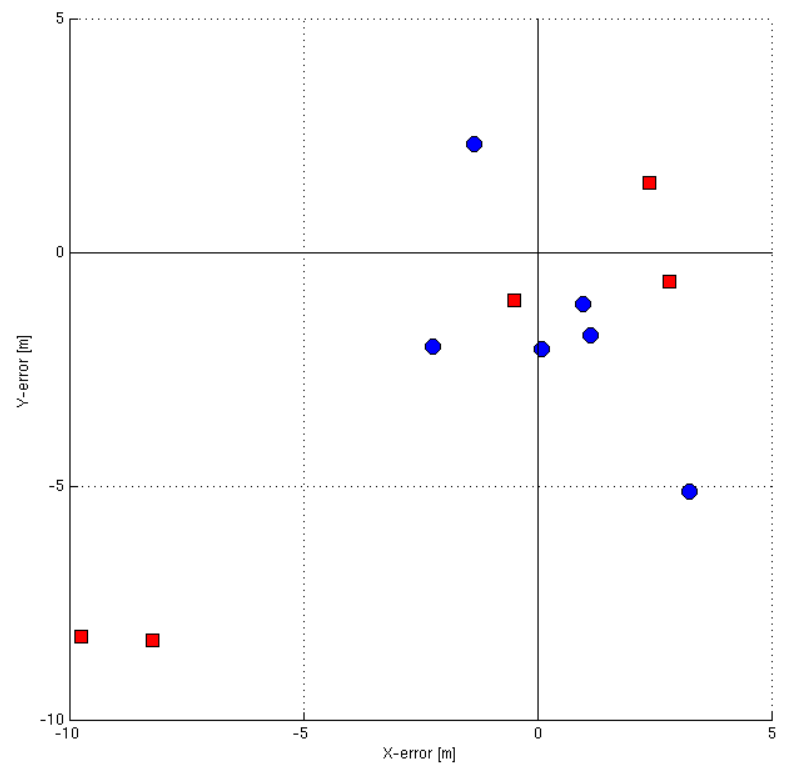

Figure 8: Return position errors in the vicinity of the target point $(0,0)$ after walking fast along the 2-D closed-loop path in either: (O-blue color) CW or ( $\square$-red color) CCW direction.

5. The total travel distance in the fast-walk experiment is slightly shorter because the subject approximated the rectangular path with a larger turning radius at the corners.
Table 4: Summary of return position errors for the CW 2-D closed-loop path experiment (fast walk).

\begin{tabular}{|l|c|c|c|c|c|}
\hline & $\begin{array}{c}\boldsymbol{X}_{e} \\
{[\mathbf{m}]}\end{array}$ & $\begin{array}{c}Y_{e} \\
{[\mathbf{m}]}\end{array}$ & $\begin{array}{c}\boldsymbol{D} \\
{[\mathbf{m}]}\end{array}$ & $\begin{array}{c}E_{a} \\
{[\mathbf{m}]}\end{array}$ & $\begin{array}{c}E_{r} \\
{[\%]}\end{array}$ \\
\hline Walk 1 & 0.08 & -2.08 & 521 & 2.08 & 0.4 \\
\hline Walk 2 & -1.36 & 2.32 & 511 & 2.69 & 0.53 \\
\hline Walk 3 & 3.23 & -5.12 & 513 & 6.05 & 1.18 \\
\hline Walk 4 & 0.96 & -1.12 & 519 & 1.47 & 0.28 \\
\hline Walk 5 & 1.12 & -1.78 & 518 & 2.1 & 0.41 \\
\hline Average & & & $\mathbf{5 1 6}$ & $\mathbf{2 . 8 8}$ & $\mathbf{0 . 5 6}$ \\
\hline
\end{tabular}

Table 5: Summary of return position errors for the CCW 2-D closed-loop path experiment (fast walk).

\begin{tabular}{|l|c|c|c|c|c|}
\hline & $\begin{array}{c}\boldsymbol{X}_{\mathrm{e}} \\
{[\mathrm{m}]}\end{array}$ & $\begin{array}{c}Y_{\mathrm{e}} \\
{[\mathrm{m}]}\end{array}$ & $\begin{array}{c}\boldsymbol{D} \\
{[\mathrm{m}]}\end{array}$ & $\begin{array}{c}\boldsymbol{E}_{\mathrm{a}} \\
{[\mathbf{m}]}\end{array}$ & $\begin{array}{c}\boldsymbol{E}_{r} \\
{[\%]}\end{array}$ \\
\hline Walk 1 & -0.5 & -1.02 & 516 & 1.14 & 0.22 \\
\hline Walk 2 & 2.81 & -0.63 & 513 & 2.88 & 0.56 \\
\hline Walk 3 & -8.23 & -8.31 & 514 & 11.7 & 2.28 \\
\hline Walk 4 & 2.39 & 1.48 & 521 & 2.81 & 0.54 \\
\hline Walk 5 & -9.75 & -8.22 & 513 & 12.76 & 2.49 \\
\hline Average & & & $\mathbf{5 1 5}$ & $\mathbf{6 . 2 6}$ & $\mathbf{1 . 2 2}$ \\
\hline
\end{tabular}

\subsection{Long Walk}

In this set of experiments the subject started inside a building, walked outside, followed the curb of a cul-de-sac street and returned back to the starting point. The street was generally slightly sloped and between the building and the street was as short but steep slope of about two meters height. The subject completed each walk in about 10 minutes at normal walking speed. Because of the longer duration of these walks, the potentially large bias drift of the nIMU introduced larger heading errors in some of the walks. The large heading error resulted in correspondingly large position errors.

The subject walked this path twice in CW and twice in CCW direction. Figure 9 shows the trajectory of the best and

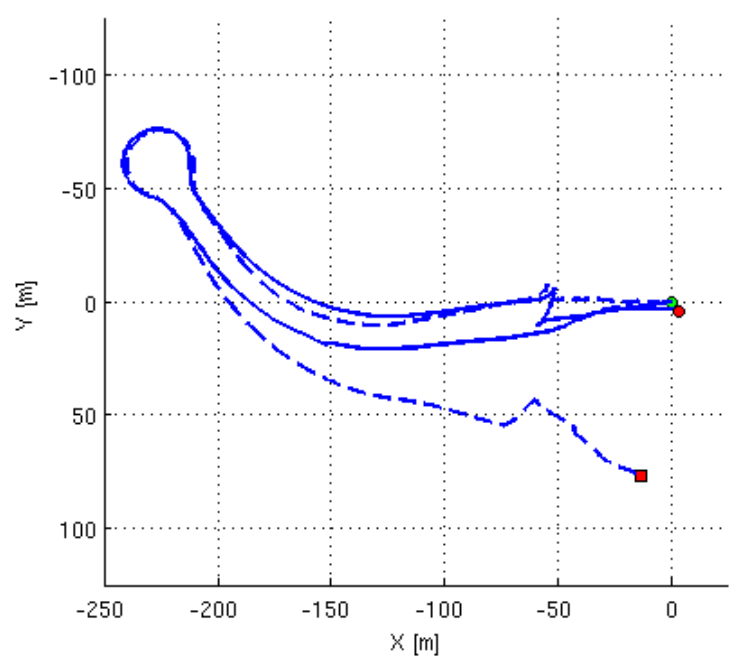

Figure 9: Trajectory of a subject on the long walk. (Solid-line): best result, (dashed-line) worst result.

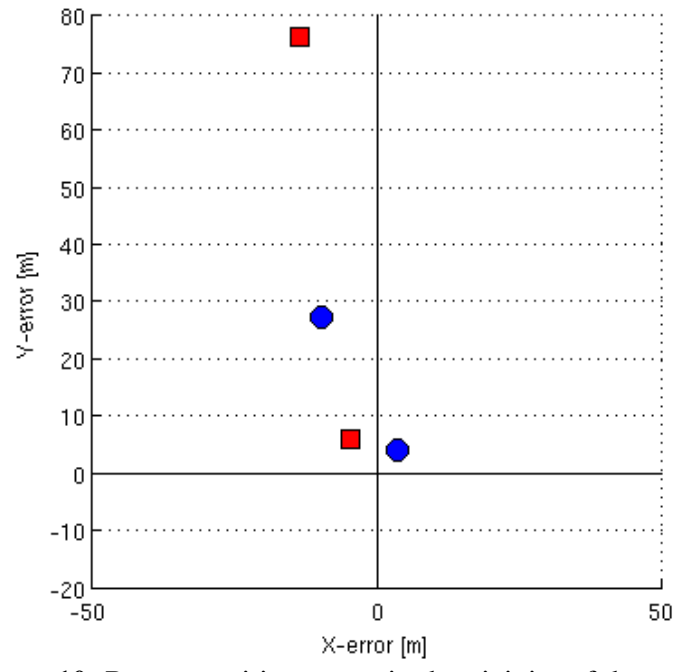

Figure 10: Return position errors in the vicinity of the target point $(0,0)$ after the long walk: (O-blue color) CW or ( $\square$-red color) CCW direction. 
worst walk of the total of four walks. Figure 10 shows the final position errors and Table 6 summarizes the results numerically.

\section{CONCLUSIONS}

Our earlier work with the bulky SiIMU01 proved the feasibility of the PDR concept. This paper here presents a snapshot of the current state of development of our PDR system using the small-sized nIMU. Since this project is currently ongoing, we could present in this paper only a limited set of experimental results. Nonetheless, these limited results, together with the extensive experimental results obtained with the SiIMU01 in our earlier work, suggest that the PDR system can indeed be based on a small-sized IMU, despite that nIMU's performance limitations.

As tested to date, the nIMU-based system is very accurate in measuring linear displacements (i.e., distance traveled, a measure similar to that provided by the odometer of a car), with errors being consistently less than $2 \%$ of distance traveled. The PDR system is also indifferent to pauses or changes in walking gaits. The accuracy of the PDR system degrades gracefully with extreme modes of legged locomotion, such as running, jumping, and climbing.

In the second mode of operation, dead-reckoning, the PDR system measures relative position in terms of X-Y-Z coordinates. Because of the limited performance of the gyroscopes inside the nIMU, the heading errors and the position estimation accuracy are larger than with the SiIMU01, and these errors grow faster than those of the SiIMU01 as a function of time. We are currently investigating methods for reducing these problems such as using a magnetometer or implementing a Kalman filter to estimate some of the random errors.

\section{Acknowledgements}

This material is based upon work supported by Mercury under Contract No. UM-PDR-001, as well as by the Department of the Army via sub-contract No. S-8844-UM-03 and administered by General Dynamics Corporation. Funding was also provided by the U.S. Dept. of Energy under Award No. DE FG52 2004NA25587.

\section{BibLIOGRAPHY}

[1] Butz, A. Baus, J. And Kruger A. (2000). “Augmenting buildings with infrared information.” Proceedings of the International Symposium on Augmented Reality, IEEE Computer Society Press, pp. 93-96.

[2] Cho, S.Y. and Park (C.G., 2006). "MEMS Based Pedestrian Navigation System.” This Journal, vol. 59, pp. 135153.

[3] Newman, J., Ingram, D. and Hopper A. (2001). “Augmented reality in a wide area sentient environment.” Proceedings of the IEEE and ACM International Symposium on Augmented Reality, pp. 77-86.

[4] Liu, Y., Wang, Y., Dayuan Y. And Zhou Y. (2004). "DPSD algorithm for AC magnetic tracking system.” IEEE Symposium on Virtual Environments, Human-Computer Interfaces and Measurement Systems, pp. 101-106.

[5] Kourogi M. and Kurata T. (2003). "Personal positioning based on walking locomotion analysis with self-contained sensors and a wearable camera." Proc. of the Second IEEE and ACM International Symposium on Mixed and Augmented Reality, pp. 103-112.

[6] Galindo, C. et al. (2005). "Vision SLAM in the measurement subspace.” Proceedings of the IEEE International Conf. on Robotics and Automation, Barcelona, Spain, pp. 30-35.

[7] PointResearch/Honeywell, (2006), http://pointresearch.com/products.html\#DRM, last accessed: 03/2007.

[8] Saarinen, J., Suomela, J., Heikkila, Elomaa, M., and Halme, A. (2004). “Personal navigation system.” Proceedings of the 2004 IEEE/RSJ International Conference on Intelligent Robots and Systems, vol 1, pp. 212-217. 
[9] Brand, T. And Phillips, R. (2003). “Foot-to-Foot Range Measurement as an Aid to Personal Navigation.” 59th Institute of Navigation Annual Meeting. Albuquerque, NM.

[10] Ojeda, L., Borenstein, J. "Non-GPS Navigation for Emergency Responders." 2006 International Joint Topical Meeting: Sharing Solutions for Emergencies and Hazardous Environments. Salt Lake City, UT, February 12-15, 2006.

[11] Ojeda, L., and Borenstein, J. (2007). "Non-GPS Navigation for Security Personnel and Emergency Responders.” Journal of Navigation. Vol. 60 No. 3, September 2007. In Print.

[12] BAE Systems, plc, http://www.baesystems.com, London, United Kingdom, last accessed: 04/2007.

[13] MemSense, LLC, http://www.memsense.com, Rapid City, SD, last accessed: 03/2007.

[14] Real Time Application Interface, http://www.rtai.org, Politecnico di Milano - Dipartimento di Ingegneria Aerospaziale, Italy, last accessed: 03/2007.

[15] Ojeda, L., Chung, H., and Borenstein, J. (2002). "Precision Calibration of Fiber-optics Gyroscopes for Mobile Robot Navigation." Proc. 2000 IEEE International Conference on Robotics and Automation, San Francisco, CA, pp. 2064-2069, April 24-28.

[16] IEEE Std.952-1997. (1998) "IEEE Standard Specification Format Guide and Test Procedure for Single-Axis Interferometric Fiber Optic Gyros” IEEE Standards, Piscataway, NJ, USA, 1998

[17] Titerton, D. and Westaon, J. (2004). “Strapdown Inertial Navigation Technology, 2nd Edition.” Progress in Astronautics and Aeronautics Series, Published by AIAA.

[18] Savage, P. (1998). "Strapdown Inertial Navigation Integration Algorithm Design. Part 1: Attitude Algorithms", Journal of guidance, control, and dynamics, vol. 21, no. 1, pp. 19-28

[19] Ayyappa, E. (1997). "Normal Human Locomotion, Part 1: Basic Concepts and Terminology.” Journal of Prosthetics \& Orthotics, vol. 9, no. 1, pp. 10-17.

[20] Huddle, J. (1998). “Trends in inertial systems technology for high accuracy AUV navigation.” Proceedings of the 1998 Workshop on Autonomous Underwater Vehicles. AUV'98. pp. 63-73.

[21] Ledroz, A. et al. (2005). "FOG-based navigation in downhole environment during horizontal drilling utilizing a complete inertial measurement unit: directional measurement-while-drilling surveying." IEEE Transactions on Instrumentation and Measurement, vol. 54, no. 5, pp. 1997-2006.

[22] Grejner-Brzezinska D. A., Yi Y, and Toth C. K. (2001). "Bridging GPS Gaps in Urban Canyons: Benefits of ZUPT”, Navigation Journal, vol. 48, no. 4, pp. 217-225. 